\title{
PERSPECTIVES FOR HADRONTHERAPHY
}

\author{
UGO AMALDI *
}

RIASSUNTO. - Con il termine "adroterapia" si intende la moderna tecnica di radioterapia oncologica che utilizza radiazioni adroniche per ottenere il controllo loco-regionale dei tumori. Sono dette adroni tutte le particelle non elementari fatte di quark: protoni e neutroni (ciascuno composto di tre quark di tipo $u$ e $d$ ) sono gli adroni più noti. Anche i nuclei degli atomi, sistemi legati di protoni e neutroni, sono adroni.

La cessione di energia di protoni di $200-250 \mathrm{MeV}$ in tessuto è tale che, anche con una sola direzione di incidenza, è possibile dare una dose elevata a un bersaglio tumorale che si trova a 25 - 30 centimetri di profondità risparmiando i tessuti sani circostanti, molto meglio di quanto non sia possibile fare anche con le più moderne tecniche che usano i raggi X (IMRT). Gli ioni carbonio da $4500 \mathrm{MeV}$ cedono - in ogni segmento del proprio percorso nel corpo del paziente - venti volte più energia dei protoni che giungono alla stessa profondità. Dato questo elevato trasferimento lineare di energia gli ioni hanno una maggiore efficacia biologica dei protoni e non hanno rivali nel controllo dei tumori radioresistenti. In particolare i risultati preliminari ottenuti a HIMAC (Giappone) su centinaia di tumori del polmone e del fegato sono estremamente incoraggianti.

Questo contributo si propone come primo scopo la presentazione dell'enorme sviluppo che si è avuto nell'ultimo triennio per ciò che riguarda i centri ospedalieri di adroterapia, ove con il termine "centro ospedaliero" si intende un centro che è dotato di più sale di trattamento ed è unicamente dedicato alla terapia delle malformazioni e dei tumori anche profondi.

Nella seconda parte dell'intervento sono descritti i due principali progetti: il Centro Nazionale di Adroterapia Oncologica (CNAO) in fase di avanzata realizzazione a Pavia e in cui si potranno trattare oltre 1000 pazienti con fasci sia di pro-

* University of Milano Bicocca and TERA Foundation, Italy. 
toni che di ioni carbonio a partire dal 2008, e il LIBO, il nuovo acceleratore lineare che, messo a valle di un ciclotrone da $30 \mathrm{MeV}$ utilizzato per la diagnostica medica con isotopi radioattivi, permette di accelerare protoni fino a $200 \mathrm{MeV}$ o più in modo da permettere allo stesso centro di praticare la protonterapia.

\section{CONVENTIONAL RADIOTHERAPY}

About one third of the $15^{\prime} 000$ particle accelerators running in the world are used in biomedicine: $3 \%$ are employed in nuclear medicine and $30 \%$ are devoted to radiotherapy [1]. Most of them produce $\mathrm{X}$ - rays (and are discussed in this Section) while only about twentyfive are used as sources of hadron beams (Sections 2 - 5).

As sources of radiation radiotherapists mainly use electron linear accelerators (linacs). About 5'000 such accelerators are at present treating patients world-wide. Photon beams (usually called 'X-ray beams' by medical doctors) are characterised by an exponential absorption after a maximum which, for beams having a maximum energy of $8 \mathrm{MeV}$, is reached at a depth of $2-3 \mathrm{~cm}$.

In order to selectively irradiate deep-seated tumours, radiotherapists use multiple beams usually pointing to the geometrical centre of the target. These irradiation techniques are applied by having the structure containing the linac rotate around a horizontal axis (Fig. 1).

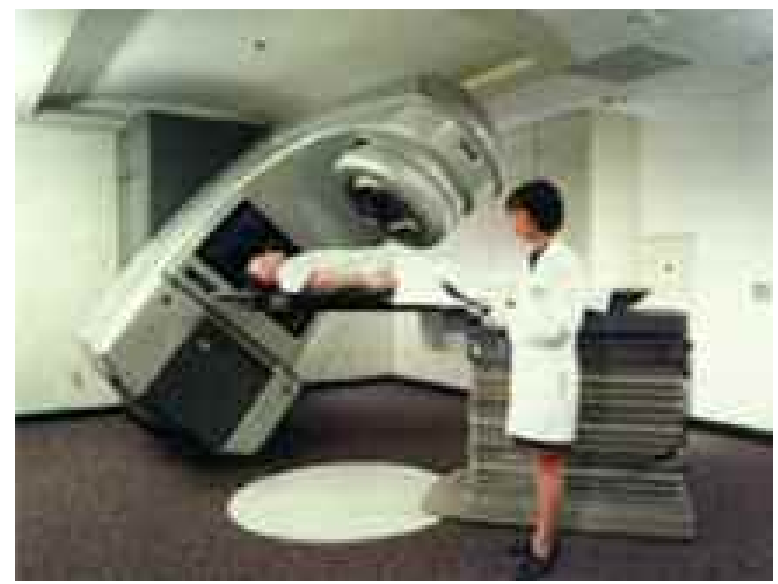

Fig. 1.

A modern $3 \mathrm{GHz}$ linear accelerator used for photon and electron treatments rotates around the patient, so that the beam can be directed to the target from any direction. 
In a typical treatment session 2-2.5 Gy are delivered to the tumour target, while delivering less than 1-1.2 Gy to any of the organs at risk (1 gray $=1 \mathrm{~J} / \mathrm{kg}$ ). Since the treatment lasts about 30 sessions, usually spread over 6 weeks, the target will eventually have received $60-75$ Gy. It has to be underlined that even a small increase of the dose is worthwhile. For a typical tumour, which is controlled with a 50\% probability, a $10 \%$ increase of the dose usually improves this probability by 15 $20 \%$, so that the control rate increases from $50 \%$ to $65-70 \%$. This is a sizeable effect since it corresponds to a reduction of the failure rate from the initial $50 \%$ to $30-35 \%$.

The unavoidable doses given to the healthy tissues are a limiting factor. By conforming the dose to the target the dose absorbed by the tumour can be increased. Thus 'conformity' is the main goal of all recent developments in cancer teletherapy. Intensity Modulated RadioTherapy (IMRT) makes use of 6-10 X-ray beams; the beams may be non-coplanar and their intensity is varied across the irradiation field by means of variable collimators ('multileaf collimators') that are computer controlled. For planning the treatments very sophisticated codes have been developed ('inverse treatment planning').

\section{Distribution of THE DOSE With BEAMS OF CHARGED HADRONS}

"Hadrontherapy" is a collective word and describes the many different techniques of oncological radiotherapy which make use of fast non-elementary particles made of quarks: protons, neutrons and light nuclei are the hadrons used to locally control many types of tumours.

Due to the unfavourable depth-dose distribution, that is very similar to that of photon beams, neutrons are becoming less interesting, though about 20 '000 patients have been treated and good results have been obtained by irradiating tumours of the salivary glands and of the the parotis.

The depth-dose curves of proton and light ion beams are completely different from those of photons and neutrons because these charged particles have little scattering when penetrating in matter and give the highest dose near the end of their range as shown in the 'Bragg peak', just before coming to rest. The first proposal to use protons and Carbon ions in radiotherapy was put forward by Bob Wilson in 1946 
[2] who suggested to obtain a conformal treatment by exploiting the Bragg peak. It is worthwhile remarking that the existence of such a peak is a direct consequence of the fact that - below about $250 \mathrm{MeV} / \mathrm{u}$ - for all light ions the kinetic energy dependence of the energy loss is well reproduced by a simple power law: K-0.82. It follows that all Bragg peaks, i.e. all energy losses plotted as a function of the residual range $r$, are approximately given by the simple formula $r-0.45(0.45=$ $0.82 / 1.82$ ). The famous peak of a mono-energetic beam of fully stripped ions - down to a residual range of $1-2 \mathrm{~mm}$ of water - is due to divergence of the function $r-0.45$ when $r$ goes to zero.

Protons and light ions are advantageous in IMHT (Intensity Modulated Hadron Therapy) because of three physical properties. Firstly, as just said, they deposit their maximum energy density in the Bragg peak at the end of their range, where they can produce severe damages to the cells while sparing both traversed and deeper located healthy tissues. Secondly, they penetrate the patient practically without diffusion. Thirdly, being charged, they can easily be formed as narrow focused and scanned pencil beams of variable penetration depth so that any part of a tumour can be accurately and rapidly irradiated. Thus, a beam of protons, or light ions, allows highly conformal treatment of deep-seated tumours with millimetre accuracy, giving minimal doses to the surrounding tissues.

The depth of the Bragg peak depends on the initial energy of the ions and its width on the energy spread of the beam that, in order to use at best the distal steep drop of the peak, should

not be larger than about $0.2 \%$. By varying the energy during the irradiation in a controlled way, one can superimpose many narrow Bragg peaks and obtain a Spread-Out Bragg Peak (SOBP). This can be achieved in two ways: the first one is based on the interposition of an absorbing material of variable thickness in the beam path; the second one is based on the modulation of the beam energy during the irradiation. This modulation is easily feasible in some accelerators as synchrotrons, but it is more difficult with others (cyclotrons).

In order to reach in soft tissues depths of more than $25 \mathrm{~cm}-$ necessary to treat deep-seated tumours - proton and carbon ion beams must have an initial energy not lower than $200 \mathrm{MeV}$ and 4'500 MeV respectively (i.e. $375 \mathrm{MeV} / \mathrm{u}$ ).

Radiotherapists use rotating linacs to treat patients with X-ray beams (Fig. 1) and would like to have the same possibility when using proton (and ion) beams. The magnetic rigidity of $200 \mathrm{MeV}$ protons is 
such that the magnetic channel capable of doing so has a typical total radius of $4-5 \mathrm{~m}$. For this reason, fixed (mainly horizontal) proton beams have been used world-wide till 1992, when the first hospital based centre became operational at the Loma Linda Medical Centre (Los Angeles) (Fig. 2).

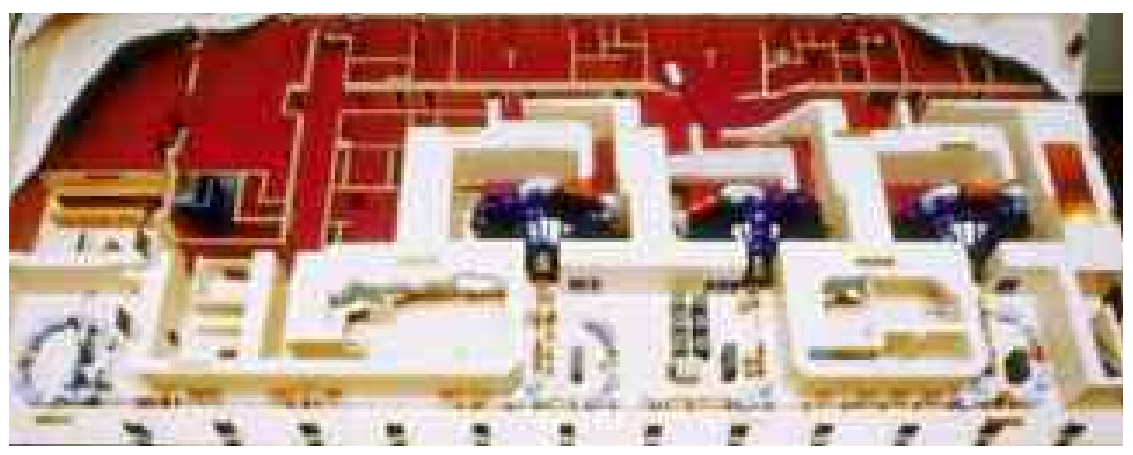

Fig. 2.

The heart of Loma Linda Medical Center is a $7 m$ diameter synchrotron built by Fermilab. The protons are accelerated up to $250 \mathrm{MeV}$. Three gantry rooms and one room with horizontal beams are used. Passive spreading systems are mounted on all the lines.

Since then the new facilities have usually more gantries, which are large mechanical structures which rotate around a horizontal axis and rigidly support the needed bending magnets and quadrupoles.

Relatively simple 'passive spreading systems' have been used in all centres till 1997. In this approach, the protons are diffused by a first 'scatterer' and their energy is adapted to the distal form of the tumour by using appropriate absorbers. The transverse form of the irradiation field is defined by collimators. Only in 1997 at PSI (Villigen - Switzerland) the first rotating gantry with a $250 \mathrm{MeV}$ proton beam came into operation (Fig. 3).

At PSI a novel active spreading system has been implemented: the target is subdivided into many thousands of voxels and each one is irradiated in successive steps by sending the proton beam of about $5 \mathrm{~mm}$ section with a given energy and direction. Till summer 2001 about 80 patients have been treated successfully. As discussed in Section 5.2, in the same years an active spreading system of a different design was started on the medical ion beam of GSI (Darmstadt). The new hadrontherapy facilities will all have the possibility of treating patients with active spreading systems. 


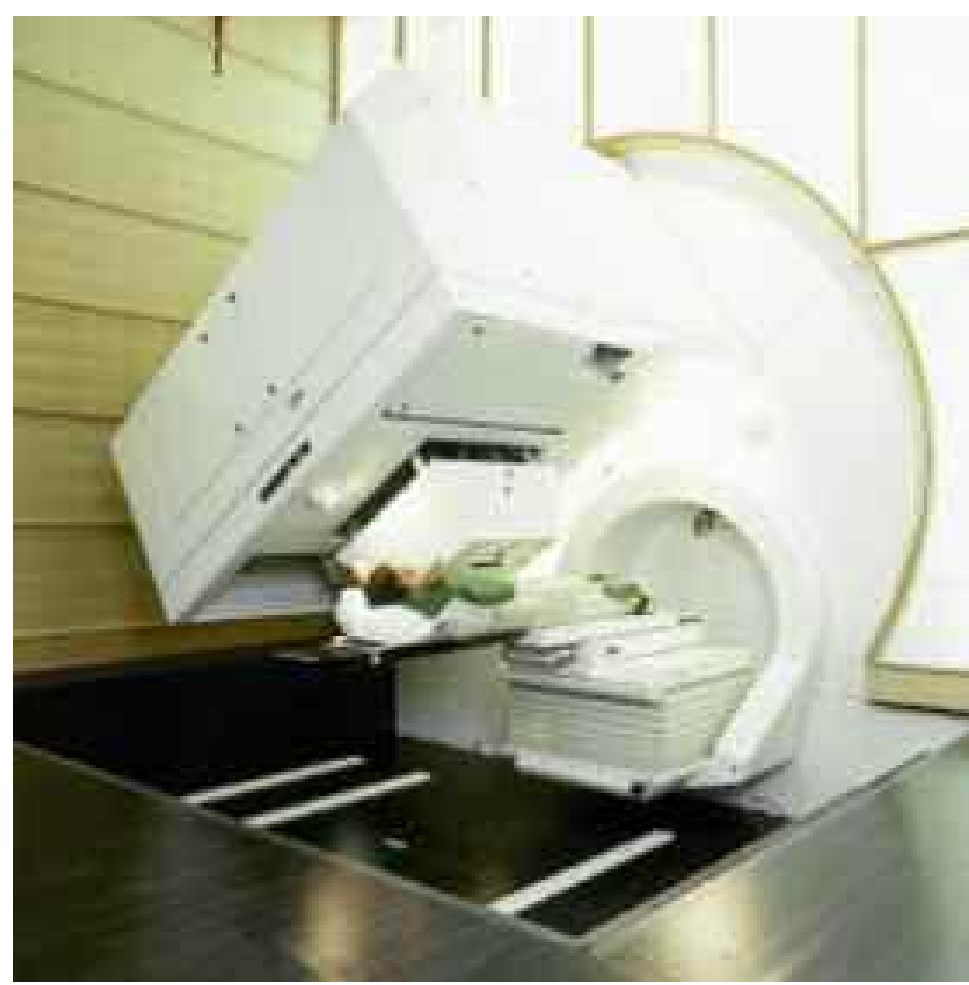

Fig. 3.

The PSI rotating gantry features a novel system for distributing the dose called "spot scanning" [3].

For eye melanoma, as for the treatment of macular degeneration, protons of energies in the range $60-70 \mathrm{MeV}$ are enough and passive spreading is sufficient: the accelerators, which are listed in Table 1, are much smaller and there is no need for active dose distribution. Contrary to deep therapy, this type of treatment is well developed in Europe. The most important centres are at the PSI, the Centre Antoine Lacassagne (Nice, France), the Clatterbridge Centre for Oncology (UK), the Centre de Protonthérapie d'Orsay (France) and the Lisa Meitner Centre in Berlin. In October 2001 patients will be treated at the Laboratori Nazionali del Sud (LNS) of INFN in Catania, where an external source has been added to the superconducting cyclotron running since many years. 
Table 1.

Number of patients irradiated with protons till summer 2001[4].

\begin{tabular}{|c|c|c|c|c|c|c|c|c|}
\hline Centre & $\begin{array}{c}\text { First } \\
\text { therapy }\end{array}$ & $\begin{array}{l}\text { Last } \\
\text { therapy }\end{array}$ & Accel & Beam & $\begin{array}{c}\text { Max } \\
\text { energy } \\
{[\mathrm{MeV}]}\end{array}$ & $\begin{array}{c}\text { Clinical } \\
\text { energy } \\
{[\mathrm{MeV}]}\end{array}$ & $\begin{array}{c}\text { N. } \\
\text { of } \\
\text { patients }\end{array}$ & $\begin{array}{c}\text { Date } \\
\text { of } \\
\text { total }\end{array}$ \\
\hline LBL, Berkeley (USA) & 1954 & 1957 & S & Horiz. & & $70-230$ & 30 & \\
\hline GWI, Uppsala (Sweden) & 1957 & 1976 & $\mathrm{C}$ & Horiz. & 185 & $15-185$ & 73 & \\
\hline HCL, Cambridge (USA) & 1961 & & $\mathrm{C}$ & Horiz. & 160 & 160 & 8906 & July 01 \\
\hline JINR, Dubna (Russia) & 1967 & 1974 & S & Horiz. & 680 & $70-200$ & 84 & \\
\hline ITEP, Moscow (Russia) & 1969 & & S & Horiz. & 1000 & $70-200$ & 3414 & June 01 \\
\hline LINPH, St. Petersburgh (Russia) & 1969 & & SC & Horiz. & 1000 & 1000 & 1029 & June 98 \\
\hline NIRS, Chiba (Japan) & 1979 & & $\mathrm{C}$ & Horiz. & 90 & $70-90$ & 133 & Apr. 00 \\
\hline PMRC, Tsukuba (Japan) & 1983 & & $S$ & Vert. & 500 & $\leq 250$ & 700 & July 00 \\
\hline PSI, Villigen (Switzerland) & 1984 & & $\mathrm{C}$ & Horiz. & 590 & $\leq 250$ & 3360 & July 00 \\
\hline JINR, Dubna (Russia) & 1987 & & SC & Horiz. & 680 & $70-200$ & 88 & May 01 \\
\hline TSL, Uppsala (Sweden) & 1989 & & $\mathrm{C}$ & Horiz. & 200 & $45-200$ & 300 & June 01 \\
\hline Douglas U. Clatterbridge (UK) & 1989 & & $\mathrm{C}$ & Horiz. & 62 & 62 & 1033 & Dec 00 \\
\hline LLUMC Loma Lindan(USA) & 1990 & & $\mathrm{C}$ & $\begin{array}{c}\text { Hor. }+3 \\
\text { gant. }\end{array}$ & 250 & $70-250$ & 6174 & June 01 \\
\hline UCL, Louvain (Belgium) & 1991 & & $\mathrm{C}$ & Horiz. & 90 & 90 & 21 & Nov. 93 \\
\hline CAL, Nice (France) & 1991 & & $\mathrm{C}$ & Horiz. & 65 & 65 & 1590 & Jun. 00 \\
\hline $\mathrm{CPO}$, Orsay (France) & 1991 & & SC & Horiz. & 200 & $73-200$ & 1894 & Jan. 01 \\
\hline NAC, Faure (South Africa) & 1993 & & $\mathrm{C}$ & Horiz. & 200 & $\leq 200$ & 398 & June 01 \\
\hline IUCF, Indiana (USA) & 1993 & & $\mathrm{C}$ & Horiz. & 200 & $75-200$ & 34 & Dec. 99 \\
\hline UC Davis, Calif. (USA) & 1994 & & $\mathrm{C}$ & Horiz. & 200 & $\leq 200$ & 284 & June 99 \\
\hline TRIUMF (Canada) & 1995 & & $\mathrm{C}$ & Horiz. & & 70 & 57 & June 00 \\
\hline PSI, Villigen ( Switzerland) & 1996 & & $\mathrm{C}$ & Gantry & 800 & 250 & 72 & Dec. 00 \\
\hline Berlin (Germany) & 1998 & & $\mathrm{C}$ & Horiz. & 65 & 65 & 166 & Dec. 00 \\
\hline NCC, Kashiwa ( Japan) & 1998 & & $\mathrm{C}$ & $\begin{array}{c}\text { Hor. }+2 \\
\text { gant. }\end{array}$ & 235 & 235 & 75 & May 01 \\
\hline \multicolumn{7}{|c|}{ TOTAL } & 29.852 & \\
\hline
\end{tabular}

In the following only hospital-based centres having more than one irradiation room devoted to hadrontherapy of deep-seated tumours will be discussed. Typically in such a centre between 10'000 and 20'000 irradiation sessions are held every year.

To date about 30'000 patients have undergone protontherapy (Table 1) and, as far as deepseated tumours are concerned, very good results have been obtained in head and neck cancers. Clinical data on protontherapy, indications, protocols and results are summarised in Ref. [5]. 
Many protontherapy protocols are well defined and phase III trials are under way to accurately compare the results with those of conventional radiotherapy. By summarising a long chain of arguments, one can state that most experts agree on the fact that protontherapy is a better treatment that even the best Intensity Modulated Radiation Therapies for about $1 \%$ of all the patients irradiated nowadays with $\mathrm{X}$-rays. Large tumours are, in particular, elective targets because with $\mathrm{X}$-rays the surrounding tissues receive unavoidably a much larger dose. This comes out clearly from the treatment planning comparison of Fig. 4.
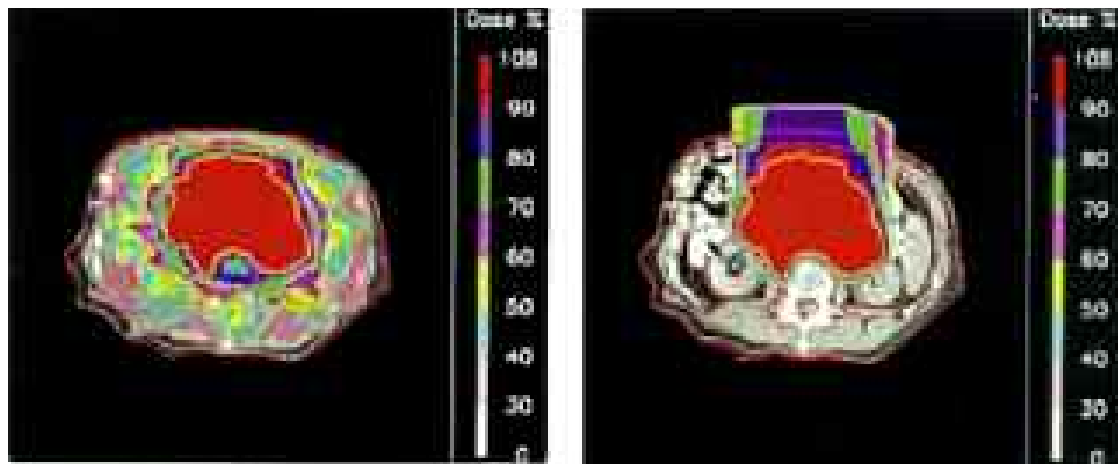

Fig. 4

The proton treatment plan shown at the right is definitely better that the IMRT treatment that uses nine X-ray beams and is plotted to the left [6]. The active proton distribution system developed at the Paul Scherrer Institute was used.

Since in a population of 10 millions Europeans about 20'000 people are irradiated every year with X-rays, this correspond to about 200 patients. Moreover, for about $10 \%$ of the usual treatments, i.e. for about 2'000 patients per year, protontherapy should give a better tumour control, but more clinical data are needed to exactly quantify the advantages.

Carbon beams of about $400 \mathrm{MeV} / \mathrm{u}$ are indicated for treatment of deep-seated tumours, which are radioresistant both to X-rays and to protons. The radiobiological arguments can be summarised as follows [7]. The dense column of ionisation produced near the Bragg peak of a light ion track gives rise to many Double Strand Breaks and Multiple Damaged Sites, when it crosses the DNA of a cell nucleus. The effects on the cell are thus qualitatively different from the ones produced by sparsely 
ionising radiations, as X-rays, electrons and protons, which interact mainly indirectly with DNA, mostly producing repairable Single and Double Strand Breaks. Due to the much larger proportion of direct effects, light ions have - for many 'end-points' and delivered doses - a Radio Biological Effectiveness (RBE), which is about three times larger than that of X-rays and protons. They are therefore suited for clinical situations where the radio resistance - linked to hypoxia or being "intrinsic" - is a difficult problem to overcome both with conventional radiation therapy and with protons. A number of results show that these qualitative differences show up at LETs larger than about $20 \mathrm{keV} / \mu \mathrm{m}$. This is physically understandable, since it corresponds to an average energy deposition of $40 \mathrm{eV}$ in the $2 \mathrm{~nm}$ thickness of a crossed DNA fibre, just what is needed to produce on average one ionisation.

About ten years ago, radiobiologists and radiotherapists have reached the conclusion that the optimal ions are found in the range $Z=$ 3 - 6, i.e. between Lithium and Carbon. There are two

main reasons for this. Firstly, LET values larger than $20 \mathrm{KeV} / \mu \mathrm{m}$ can be confined to the tumour tissues: in the case of Carbon (Lithium) this approximate threshold is passed for a residual range in water of 47 $\mathrm{mm}(5 \mathrm{~mm})$. Secondly, the part of the incoming ions fragment into lighter ones, that have a lower charge and thus a longer range than the parent ions. This effect increases with $Z$ and produces a 'tail' in the dose distribution downstream of the rapid falloff of the Bragg peak often irradiating healthy tissues.

As far as the number of patients are concerned, the starting point is that about $20 \%$ of all the tumours currently treated with X-rays are resistant, so that the number of new radioresistant tumours per year is about 4'000 on a population of 10 millions. Between one third and half of these tumour would benefit from an ion treatment, corresponding to at least 1'300 patients/year, but part of this cohort overlaps with the one of the already discussed protontherapy patients. Specific indications thus correspond to about 1000 patients/year on 10 millions inhabitants.

Since the usual cellular repair mechanisms have little effect, there is no point in fractionating the dose in the 30 sessions used with $\mathrm{X}$-rays. This shortening of the treatment is an advantage, but the lack of repair may also induce late recurrencies in the healthy tissues and requires a lot of care in modelling the Relative Biological Effectiveness of a complex irradiation field. Potential problems can be overcome by using a Carbon beam to deliver in 3-4 sessions a 'preboost' to the central part 
of hypoxic radioresistant tumours followed by a conventional treatment (20-30 sessions) with X-rays [7]. This proposal is also interesting for an effective use of the costly infrastructures to be discussed in Section 5.

Whatever treatment schedule is used, many more clinical data are needed because only 1'000 patients have been irradiated with Carbon ions world-wide. Indeed the available clinical results are mainly due to the activity pursued since 1994 in Japan at HIMAC (Heavy Ion Medical Accelerator Centre, Chiba) - where about 900 patients have been irradiated with passive spreading systems [8] - and to GSI (Darmstadt) [9]. Here pioneering work in the simulation of the RBE of ions has been done and about hundred patients have been treated with the raster scanning technique described in Section 5.1.

\section{CENTRES FOR DEEP PROTONTHERAPY}

When discussing deep protontherapy, it should be underlined that only two of the presently running facilities have been built as fully dedicated hospital based centre: the Loma Linda University Centre (Fig. 2) and the Proton Therapy Facility of NCC at Kashiwa in Japan. All the other centres make use of beams produced by accelerators built for fundamental research in nuclear and particle physics and later adapted to protontherapy. Such centres have been running, some since long time, in Euroland, Japan, Russia and USA (Table 1). In South Africa there is a well-equipped centre in FAURE (NAC), where neutrontherapy is also performed and a new proton beam line is being commissioned.

\subsection{Centres under either commissioning or construction}

In Europe deep protontherapy with charged beams is carried out in Orsay (CPO) and Uppsala at two modified nuclear physics cyclotrons. Thus the recent increase of interest in hadrontherapy throughout Europe is quite natural, as in the year 2002 there will be two dedicated hospital-based centres for deep protontherapy in the United States and four in Japan (Fig. 5).

The Loma Linda Center is represented in Fig. 2. The second hospital based centre in the States, the Northeast Proton Therapy Center in Boston of Mass General Hospital in Boston, will treat patients from 
Fall 2001. It is based on a $230 \mathrm{MeV}$ cyclotron built by the Belgian Company IBA and aims at treating 1'000 patients/year in two gantry rooms and one room with two horizontal fixed beams. In 1999 it was decided to upgrade the cyclotron of Indiana University at Bloomington. As a consequence in 2003 there will be a third American centre for deep proton therapy,the Midwest Proton Radiation Institute, featuring two horizontal beams and an isocentric gantry.

Towards the end of 2001 two Chinese private hospitals have ordered to IBA cyclotrons and gantries. The hospitals are in the towns of Zibo (Wanjie Tumor Hospital) and of Xian (Chang An Information Industry Group) and the treatments should start in 2003.

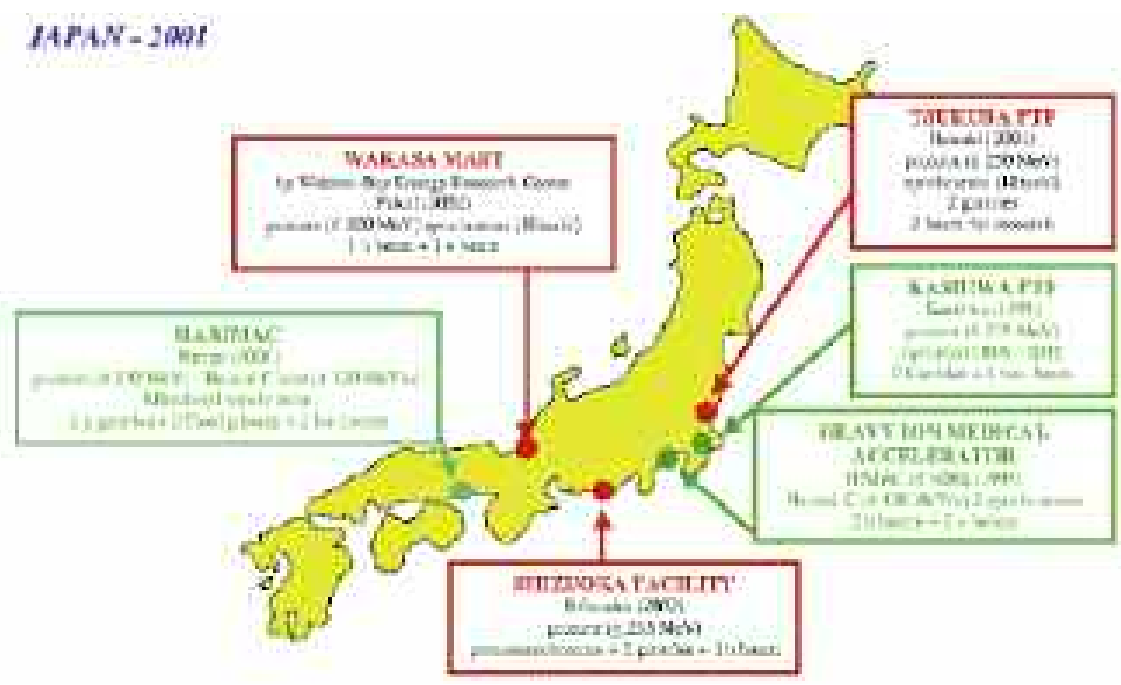

Fig. 5.

In Japan there are four protontherapy centres and two centres (HIMAC at Chiba and HARIMAC at Hyogo) featuring synchrotrons that can accelerate light ions.

They are discussed in Section 5. The centres represented in green were active in summer 2001.

In Europe a new project (PROSCAN) has been launched at the end of the year 2000 by the Paul Scherrer Institute. For it a new superconducting protoncyclotron has been ordered to ACCEL (Fig. 6). The proton beam will serve both the existing eccentric gantry and a new isocentric gantry and, to actively distribute the dose, an improved version of the PSI spot scanning technique will be implemented. 


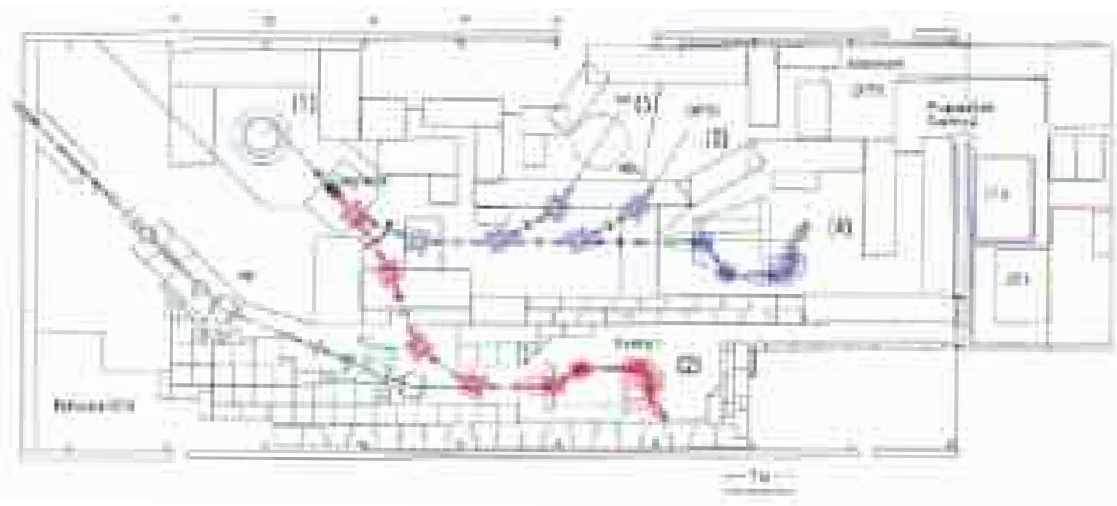

Fig. 6.

The PROSCAN project, to be built by PSI in Villigen, will feature a new cyclotron and two rotating gantries delivering protons with the spot scanning technique [10].

The company ACCEL has already sold a superconducting cyclotron to Probealth $A G$ near Munich. The Rinecker Proton Therapy Centre (RPTC) will have a multi-purpose treatment room and four gantries.

\subsection{Linear accelerators for protontherapy}

Usually proton linear accelerators run at low frequencies, have diameters of the order of one meter and accelerate large currents. Since the currents needed for protontherapy are only few nanoamperes, one can use high frequency accelerating structures, which have small apertures and large accelerating gradients. The idea of using $3 \mathrm{GHz}$ structures with gradient of the order of $15 \mathrm{MeV} / \mathrm{m}$ is at the basis of the studies initiated in 1993 by the TERA Foundation, in collaboration with ENEA (Frascati) and Istituto Superiore di Sanità (ISS, Rome) [11]. This activity has brought to the "TOP" project of ISS and to the "LIBO" project.

"TOP" stands for Terapia Oncologica con Protoni. The project is based on the construction in Rome, in collaboration with ENEA and the oncological institute IRE, of a $3 \mathrm{GHz}$ linear accelerator that will be used for eye therapy. The linac, about $10 \mathrm{~m}$ long, is based on an accelerating structure of novel design named 'Side Coupled Drift Tube Linac' [12]. 
LIBO stands for LInac BOoster. As shown in Fig. 7, it is a $13 \mathrm{~m}$ long copper structure which, installed downstream of a small cyclotron, will be capable of accelerating the protons extracted from the cyclotron from 50-70 MeV up to $200 \mathrm{MeV}$, or more. The chosen structure (Side Coupled Linac) has been designed for lower frequencies in Los Alamos, but nobody has ever used it for accelerating protons at such a high frequency.

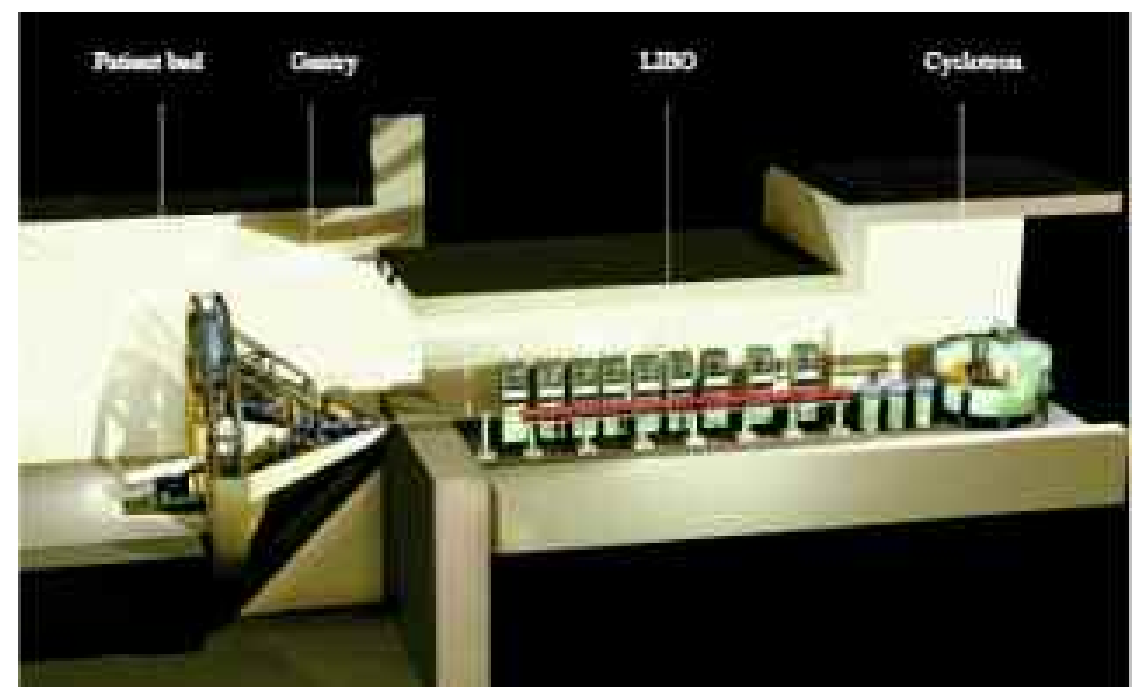

Fig. 7.

The current of a cyclotron is large (50-100 $\mu A)$ compared with what is needed for protontherapy $(10 \mathrm{nA})$. The acceptance of LIBO can thus be small and still provide the necessary beam for deep protontherapy. The repetition rate is $400 \mathrm{~Hz}$, well suited for a voxel active spreading system as in PSI.

In 1998 a collaboration among CERN, the Universities and INFN Sections of Milan and Naples and the TERA Foundation was set up to construct a $1.25 \mathrm{~m}$ long module of LIBO capable of accelerating protons from 62 to $74 \mathrm{MeV}$. The module - power tested at CERN at the end of the year 2000 - performs better than foreseen, since in each of the 4 tanks the gradient is $27 \mathrm{MeV} / \mathrm{m}$ instead of $15.5 \mathrm{MeV} / \mathrm{m}$ [13]. In autumn 2001 - in collaboration with IBA/Scanditronix - an acceleration test (from 62 to $74 \mathrm{MeV}$ ) will be performed in Catania at the Laboratori Nazionali del Sud of INFN. 


\section{Centres With BeAmS OF Light IONS}

In 1994 the first patient was treated at HIMAC in Japan with Carbon ions and with a passive dose distribution system. As already mentioned, at this centre about 1000 patients affected by brain glioma, tumours of the cervico-cephalic area, lung, liver, prostate and uterine cervix tumours have been treated. Very interesting results on some special tumour sites, as lungs and liver, have been obtained [8]. Due to the fact that $400 \mathrm{MeV} / \mathrm{u}$ carbon ions have a magnetic rigidity three times larger than $200 \mathrm{MeV}$ protons, the construction of rotating gantries poses many technical challenges. At HIMAC the choice was made to have horizontal and vertical beams in one treatment room. The other two rooms feature horizontal beams. Passive spreading systems are used, but active systems interesting will be implemented.

In the Hyogo Centre (Fig. 5) the first patient was treated with protons in May 2001. This centre has three rooms for protons (two of them with gantries) and two rooms for ions featuring also a vertical beam and an inclined beam.

\subsection{The GSI pilot project}

In December 1997 at the Darmstadt heavy ion laboratory, for the first time two brain tumours were treated with a carbon ion beam and an active distribution system. Three main features of this pilot facility are worth mentioning:

(i) the active 'raster' scanning system;

(ii) the fully automatic control of the GSI accelerator complex, that can be handled by an operator trained as operator of standard $\mathrm{X}$-ray equipment;

(iii) the two gamma ray detectors placed above and below the patient to determine 'on-line' the exact location and shape of the irradiated volume because, when penetrating the body, the incident carbon ions fragment into ${ }^{\circledR}+$ radioactive nuclei, mainly $11 \mathrm{C}$, which can be detected by a standard PET technique. 


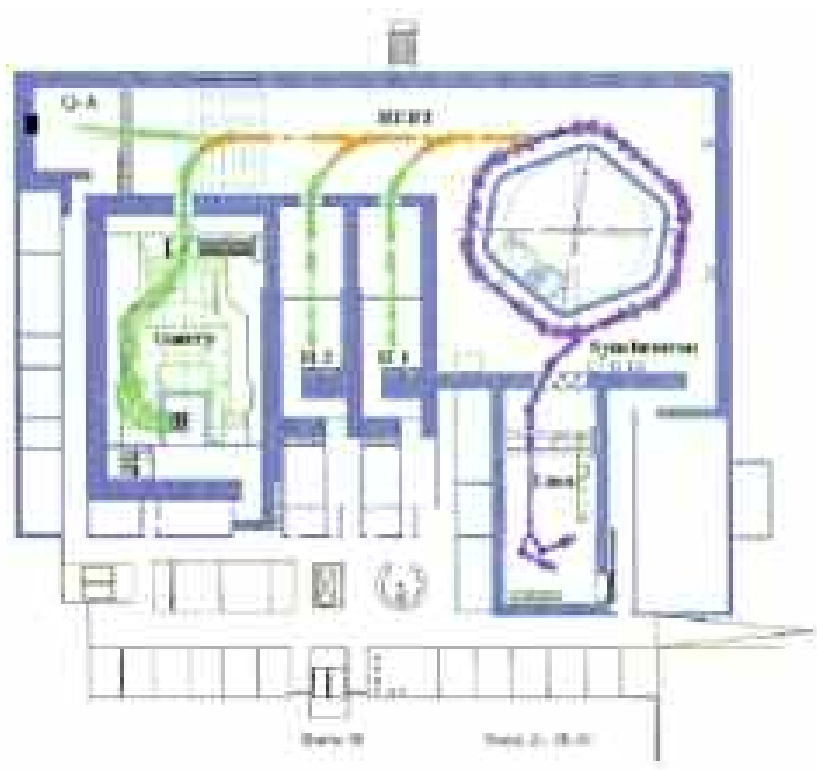

Fig. 8 .

The hadrontherapy centre proposed for Heidelberg features three treatment rooms.

One of them hosts a carbon ion rotating gantry of new design. A single $7 \mathrm{MeV} / \mathrm{u}$ linac injects in the synchrotron both protons and carbon ions [13].

The pilot project has been a great success so that about 100 patients had been treated by summer 2001. Hence, in September 1998 GSI, the oncological clinic of the University of Heidelberg and the Deutsches Krebforschungzentrum (DKFZ) have presented to the authorities the project of a hospital-based proton and ion centre capable of treating 1000 patients/year (Fig. 8). The approval is expected by october 2001.

\subsection{The Proton Ion Medical Machine Study (PIMMS)}

At the end of 1995 the TERA Foundation, together with the AUSTRON project, drew the interest of CERN on the design of an optimised synchrotron for light ion therapy, to be built by those European countries which will decide to invest the required funds. At the beginning of 1996 the CERN management agreed on the proposal and a study of such a synchroton was started at CERN under the leadership of Phil Bryant (PIMMS = Proton and Ion Medical Machine Study). 


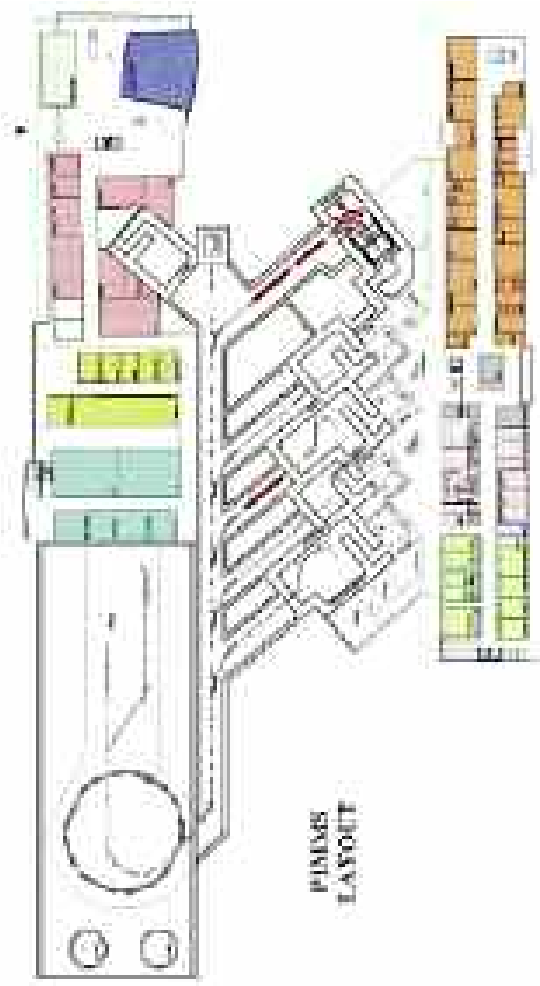

Fig. 9.

The layout of PIMMS. Protons and ions are injected by two separate linacs. The protons are used in three rooms and the carbon ions in two rooms. One of them is equipped with a 'Riesenrad' gantry. The centre covers about $12000 \mathrm{~m}^{2}$.

PIMMS is a collaboration among CERN, Med-AUSTRON (Austria), Oncology 2000 (Czeck Republic) and TERA (Italy). GSI experts have brought their competence in the many meetings held in the years 19961999 and some of them have been members of the Project Advisory Committee. The study group has published two general volumes [15] and many detailed articles.

For hadrontherapy with active spreading the main issue is the time uniformity of the spill since, mainly due to the magnet ripple, synchrotrons have time structures at many frequencies. This complicates the accurate measurement of the dose during the active spreading of the clinical pencil beams. Thus the PIMMS synchrotron has been designed starting from the clinical beam and going 'backwards'. Its extraction is based 
on a special optics and, during extraction, all magnet currents are kept constant and the energy of the circulating particles is slowly increased by the induced electric field due to a 'betatron core'.

The PIMMS layout is shown in Fig. 9. It has to be underlined that the mandate of the group was to design an optimised centre without considering budget and space constraints. The idea was that the European groups interested in construction their iontherapy centre could adapt the PIMMS proposal to their needs and budget.

In 1998 the Med-AUSTRON project, which consists in the realisation of a proton and carbon ion centre at Wiener Neurstadt in the South of Austria, proposed to the Austrian authorities a staged construction of the PIMMS project [16]. The staging allows an initial investment that is smaller than the about 120 MEuros needed for the construction of the centre of Fig. 9.

The Italian TERA Foundations aims at the construction in Italy of CNAO, the National Centre for Oncological Hadrontherapy, a healthcare and research facility equipped with both proton and ion beams so to allow the treatment of more than 1'000 patients per year. In 1997 a first proposal was presented to the Italian authorities [17].

In the years 1998-2000 a new design was prepared, based on the PIMMS synchrotron. To reduce the overall cost the surface covered by the two-floor underground bunker was reduced to $2900 \mathrm{~m}^{2}$ and a single injector was used for injecting both protons and ions. As shown in Fig. 10, the injector was located inside the synchrotron and a very compact configuration of the beam line was chosen.

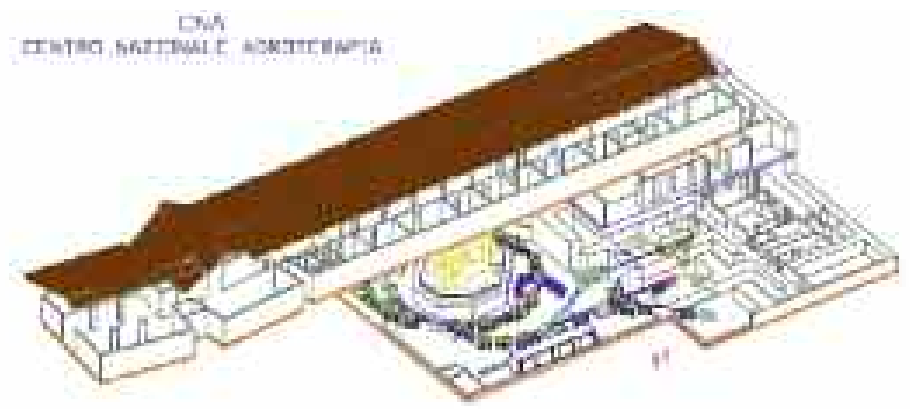

Fig. 10 .

The design of the PIMMS synchrotron has been implemented in the Italian CNAO.

Phase 1 will feature three treatment rooms for protons and ions, the central one being equipped with a horizontal and a vertical beam. In Phase 2 two gantries will be installed in an extension of the underground bunker. 
For the $7 \mathrm{MeV} / \mathrm{u}$ injector the GSI was adopted (Fig. 8). To construct the Phase 1 of CNAO 60 Meuros are needed. In 2001 the Italian Ministery of Public Health has allocated 20.6 MEuros to a Foundation that has been created to construct the centre. The board of the CNAO Foundation is formed by the directors/presidents of two University Hospitals (Milan and Pavia), of an oncological Institute (European Institute of Oncology), of the National Institute of Neurology and of TERA.

The PIMMS design is at the core of other hadrontherapy projects. In 1998 the Université Claude Bernard of Lyon asked TERA to produce a preliminary design of a Centre for Carbon ions. In summer 2000 the Claude Bernard University has signed a contract with IN2P3 and CEA with the aim of having a preliminary proposal ready by autumn 2001 . In spring 2001 the project group decided that the proposal will be based on the PIMMS synchrotron and that, as proposed by TERA, the GSI $7 \mathrm{MeV} / \mathrm{u}$ injector will be used.

In 1999 scientists from the Karolinska Institute and Hospital in Stockholm and from TERA decide to prepare together a proposal for a light ion facility to be built very close to the radiotherapy department of the Karolinska Hospital. A recent report [7] explains the rationale of such a centre and contains the plan shown in Fig. 11.

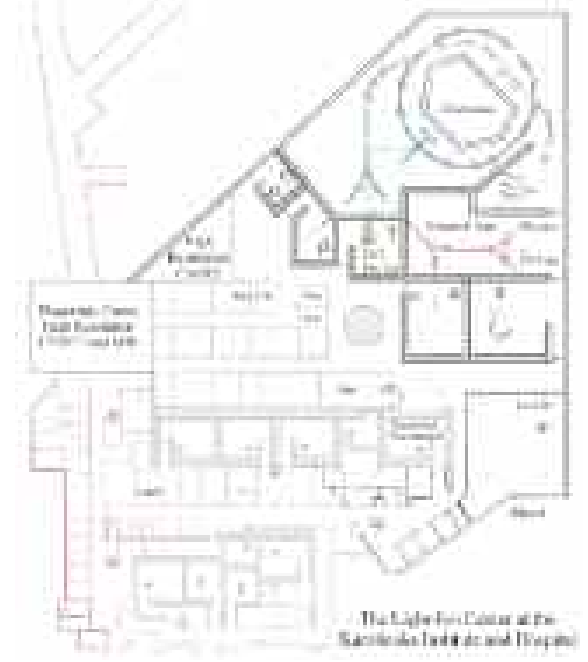

Fig. 11.

The Karolinska centre for light ions will be contiguous to the existing radiotherapy department, that features seven electron linacs and a $50 \mathrm{MeV}$ microtron used for deep therapy in four rooms [7]. 


\section{CONCLUSIONS}

Since 1998, when a similar review was published [18], many hadrontherapy centres either started treating patients or have been proposed to the local authorities. Japan is the country in which this novel radiotherapy technique is more wide-spread, but recently in Europe the interest has being growing also as a consequence of the collaboration established among nuclear research laboratories and oncological hospitals*.

\section{REFERENCES}

[1] U. Amaldi, The importance of particle accelerators, Europhysics News 31/6 (2000) 5.

[2] R.R. Wilson, Radiobiology, 47 (1946) 487.

[3] E. Pedroni, R. Bacher. H. Blattmann, T. Böhringer, A.Coray, A. Lomax, S. Lin, G. Munkel, S. Scheib, U. Schneider, and A. Tourosvsky., The 200 $\mathrm{MeV}$ proton therapy project at the Paul Scherrer Institute: conceptual design and practical realisation, Med. Phys.22 (1), January 1995.

[4] J. Sisterson (ed.), Particles, Newsletter n.27, (January 2001).

[5] R. Orecchia, A. Zurlo, A. Loasses, M. Krengli, G. Tosi, S. Zurrida, P. Zucali e U. Veronesi, Particle beam therapy (hadrontherapy): basis for interest and clinical experience, European Journal of Cancer 34 (1998) 456.

[6] A. Zurlo; A. Lomax, A. Hoess,T. Bortfeld, M. Russo, G. Goitein, V. Valentini, L. Marucci, R. Capparella, A. Loasses, The role of proton therapy in the treatment of large irradiation volumes: A comparative planning study of pancreatic and biliary tumours, Int J Radiat Oncol Biol Phys, 48: 77-288, 2000.

[7] A. Brahme, R. Lewensohn, U. Ringborg, U. Amaldi, F. Gerardi and S. Rossi, Design of a centre for radiobiologically optimised ligh ion therapy in Stockholm, TERA 01/2 GEN 30, Nucl. Inst. and Meth., in press.

[8] K. Kawachi, T. Kanai, H. Matsuzawa, T. Inada, Three dimensional spot beam scanning method for proton conformation radiation therapy, Acta Radiol. Suppl. 364 (1983) 81.

[9] J. Mizoe, H. Tsujii, T. Kamada et al, Preliminary results of Carbon-ion radiotherapy for advanced head and neck cancer, Int. Journal Rad. Onc. Bio. Phys. 46 (2000) 691.

* The first hadrontherapy center in Europe is functioning on patients in Heildenberg since 2009. The second one has been completed by CNAO in Pavia and the first patient has been treated on September 2011. 
[10] G. Kraft, The radiobiological and physical basis for radiotherapy with protons and heavier ions, Strahlenther. Onkol. 166 (1990) 10.

[11] T. Haberer, W. Becher, D. Schardt, G. Kraft, Magnetic scanning system for heavy ion therapy, Nucl. Instr. and Meth. A330 (1993) 296.

[12] J. Debus, T. Haberer, D. Schulz-Ertner, O. Jäkel, F. Wenz, W. Enghardt, W. Schlegel, G. Kraft, M. Wannenmacher, Fractionated Carbon ion irradiation of skull base tumors at GSI - First clinical results and future perspectives, Strahlenther. Onkol. 2000, 176 (5), 211-216.

[13] E. Pedroni, T. Böhringer, A. Coray, G. Goitein, M. Grossmann, A. Lomax, S. Lin, M. Jermann, A novel gantry for proton therapy at the Paul Scherrer Institute, Conf. Proceed. “Cyclotrons 2001”, East Lansing MI, May 12-17, 2001, in print.

[14] U. Amaldi, M. Grandolfo, and L. Picardi (eds.), The RITA network and the design of compact proton accelerators, INFN-Laboratori Nazionali, Frascati, (1996).

[15] L. Picardi, C. Ronsivalle, A. Vignati, Progetto del TOP linac, ENEA, Dip. Innovazione, RT/INN/97/17, (1997).

[16] B. Szelezs, U. Amaldi, P. Berra, C. Cicardi, K.R. Crandall, D. Davino, C. De Martinis, D. Giove, M.R. Masullo. E. Rosso, D. Toet, V.G. Vaccaro, M. Vretenar, M. Weiss, R. Zennaro, Successful high power test of a proton linac booster (LIBO) prototype for hadrontherapy, proceedings of PAC2001, Chicago, June 2001.

[17] Proposal for a dedicated ion beam facility for cancer therapy, J. Debus, K.D.Grass and M. Pavlovic (eds.), GSI, Darmstadt (1998).

[18] L. Badano, M. Benedikt, P.J. Bryant, M. Crescenti, P. Holy, P. Knaus, A. Meier, M. Pullia and S. Rossi, Proton-Ion Medical Machine Study (PIMMS) - Part I, CERN/PS 99-010 DI (March 1999).

[19] L. Badano, M. Benedikt, P.J. Bryant, M. Crescenti, P. Holy, P. Knaus, A. Meier, M. Pullia e S. Rossi, Proton-Ion Medical Machine Study (PIMMS) - Part II, CERN/PS 00-007 DR (July 2000).

[20] Med-Austron - Ein Österreichisches Krebsforschungs-und Behandlungszentrum zur Hadrontherapie in Europa, R. Pötter, T. Auberger and M. Regler (eds.), Verein AUSTRON, Wiener Neuerstadt (1998).

[21] The National Centre for Oncological Hadrontherapy at Mirasole, U. Amaldi (ed.), INFNLaboratori Nazionali, Frascati (1997).

[22] U. Amaldi, Cancer Therapy with Particle Accelerators, Nucl. Phys. A654 (1999) 375c.

[23] The path to the italian national centre for ion therapy, U. Amaldi and G. Magrin (eds.), Tera fnd (2010). 